\title{
Optical System for Bispectral Imaging in Mid-IR at 1000 Frames per Second
}

\author{
Marija Strojnik and Gonzalo Paez \\ Infrared Group, Research Directorate, Optics Research Center, P.O. Box 1-948, 37000 Leon, GTO, Mexico \\ Correspondence should be addressed to Marija Strojnik; mstrojnik@aol.com
}

Received 9 August 2012; Accepted 27 November 2012

Academic Editor: Carlo Corsi

Copyright (C) 2013 M. Strojnik and G. Paez. This is an open access article distributed under the Creative Commons Attribution License, which permits unrestricted use, distribution, and reproduction in any medium, provided the original work is properly cited.

We propose, evaluate, and demonstrate the performance of an IR/optical double-image experimental setup where we capture two simultaneous images of a single object, in two different spectral bands, using a single detector array. With this arrangement, we may observe rapidly changing phenomena, at a rate of more than 1000 frames per second, without the loss of the spatial information about the test subject. We describe the optical system to perform simultaneous imaging in IR for slightly inclined optical axes. We verify the actual performance by applying the experimental method to flame analysis in the mid-IR to determine the combustion efficiency.

\section{Introduction}

Several methods to measure the combustion efficiency have been presented recently to decrease the amount of undesirable by-products and utilize prudently a nonrenewable resource. Among them, we may mention the infrared (IR) cameras to detect the detailed temporal evolution [1] and interferometric techniques to assess the airflow and heat travel throughout the flame volume [2]. The applications with IR cameras allow studying the flame evolution; however, they only record the nontransparent imaginary surface within the flame volume that emits the radiation. Another shortcoming of this technique is that the combustion is a volume effect [3]. The radiation has to travel through the volume where some of its components are attenuated. Furthermore, combustion is time- and position-dependent phenomenon, even after the apparent steady state has been achieved. Another technique that has been successfully implemented to measure the temporal dependence of an established flame is the lateral shearing interferometry. The fringe distribution at the time of its capture indicates the flow of the heated gases.

For many applications, the spatiotemporal information is even more important than the spectral one. Some multispectral imaging techniques already exist [4]. However, they require expensive and sophisticated equipment for the acquisition, storage, and processing of data. They include, for example, hyperspectral imaging, available for the Earth, the ocean, and the atmospheric monitoring from mobile platforms [5]. Their usage restricts or even limits the frame rates and introduces novel focal plane layouts [6]. They often incorporate motion as a design parameter, with carefully controlled velocity vector [7]. An alternate approach has been to redistribute the spatial information into the optical fibers, and then to analyze each fiber individually [8]. This is a flexible light conduct to move image to the desired location. However, it imposes performance limitations on the achievable image resolution, while requiring a relatively elaborate experimental arrangement.

Most imaging systems capture a narrow wavelength-band image. Its width is determined by the characteristics of the optical system transmission, the detector sensitivity, and, of course, the spectral features of the object under investigation. Valuable information about a process of interest may often be obtained in just two wavelength bands. The design simplicity compensates for the loss of multispectral imaging capability. This tradeoff allows for the incorporation of additional features, as, for example, the possibility of recording detailed temporal information for the analysis of image evolution. Also, the elegance of the bispectral analysis may be of great value in routine applications of optics in medicine, including 
remote thermometry [9], and blood oximetry [10]. Bispectral techniques have been successfully applied in the areas that do not require imaging. Recently, interest in developing dualband IR detector arrays has been making excellent progress $[11,12]$.

A traditional method of recording bispectral images involves taking images of an object in succession, upon changing a filter each time $[13,14]$. This technique limits the procedure to capturing relatively slow phenomena where temporal changes take place at a rate slower than the time to change the filter. Likewise, a relatively slow bispectral technique has been reported in the evaluation of aircraft models. Similarly, in microscopy a beam splitter may be inserted inside the instrument to generate two images in two planes [15].

In this experiment we are interested in determining the combustion efficiency throughout the volume. We propose to measure the relative quantities of two combustion byproducts, within their characteristic spectral windows. This requires simultaneous measurement of transmitted radiation in two spectral channels. In this paper, we describe the optical system to achieve concurrent imaging in two IR spectral bands with precise point-to-point registration in the focal plane to allow taking ratio of detected radiation integrated over the corresponding pixel locations.

First, we lay out the preliminary experimental setup that conceptually accomplishes the bi

spectral imagery. This also serves to identify the performance requirements outlined in Section 3. On their basis the optical system is designed, accomplishing the goals. Preliminary results are presented in Section 4.

\section{Bispectral Experiment}

One of the advantages of the bispectral instrument, employed in this study, lies in measuring the relative strengths of two appropriately chosen spectral lines. Thus, only two relative measurements are needed to supply the requisite information for each pixel projected on the image plane. This makes the technique an ideal candidate for the high-speed imaging applications. We achieve bispectral imaging by capturing simultaneously two spectral images of the sample, followed by near real-time image processing, as illustrated schematically in Figure 1. We apply several algorithms to individual images. Then, we rebuild the final combined image.

With this IR/optical experimental setup we capture two images of a sample on a single detector array. They may be recorded at a rate higher than one frame per millisecond. We insert two external filters in two identical paths to overcome the limitations and possible jamming of relatively slow mechanisms. We image both onto the same detector array, but their images are spatially displaced for identification.

\section{Optical System}

In lateral or vectorial shearing interferometers, two identical beams follow "parallel paths"; while one is displaced with respect to the other, all the while remaining parallel. We

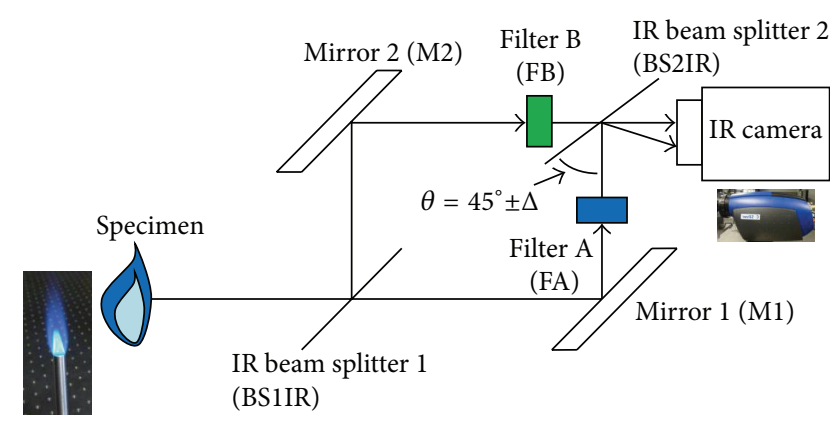

FIgURE 1: Experimental setup.

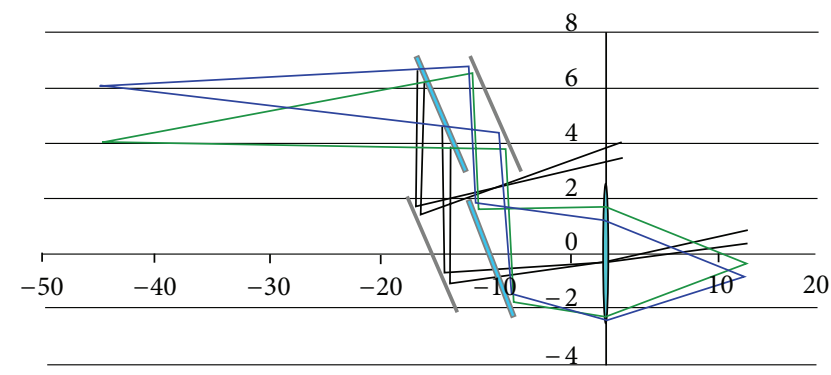

(a) 2 mirrors, 2 beam splitters

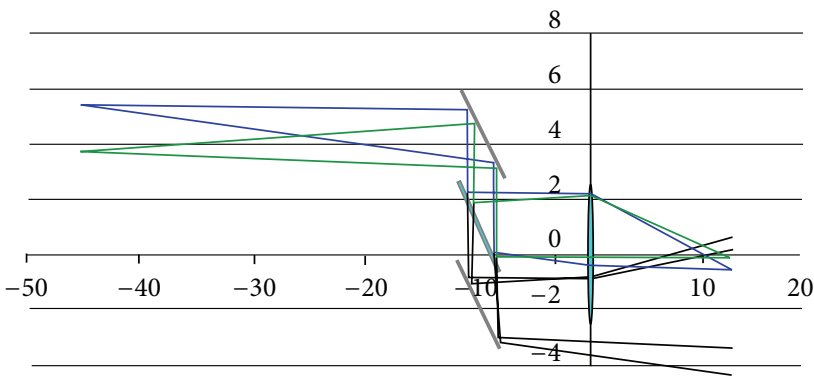

(b) 2 mirrors, 1 beam splitter

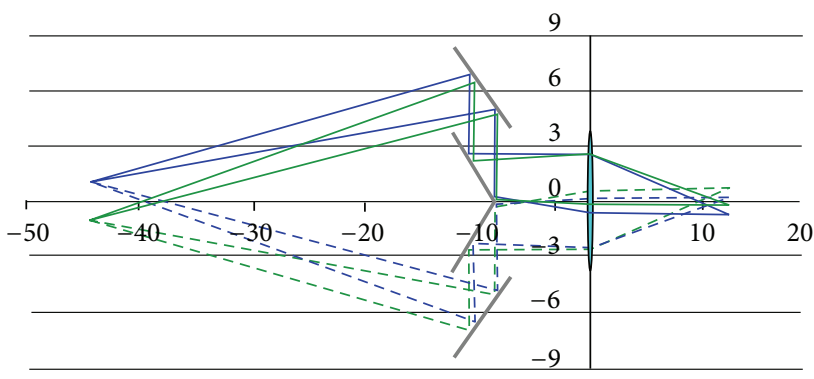

(c) 4 mirrors

FIgURE 2: Potential optical layouts to perform IR imaging in two spectral channels. The size of the instrument increases with increasing the number of mirrors.

employ a setup, somewhat similar to that known as a MachZehnder interferometer, with some critical modifications. The most important of these is the image angular displacement, similar to that in the vectorial shearing interferometer [16]. 


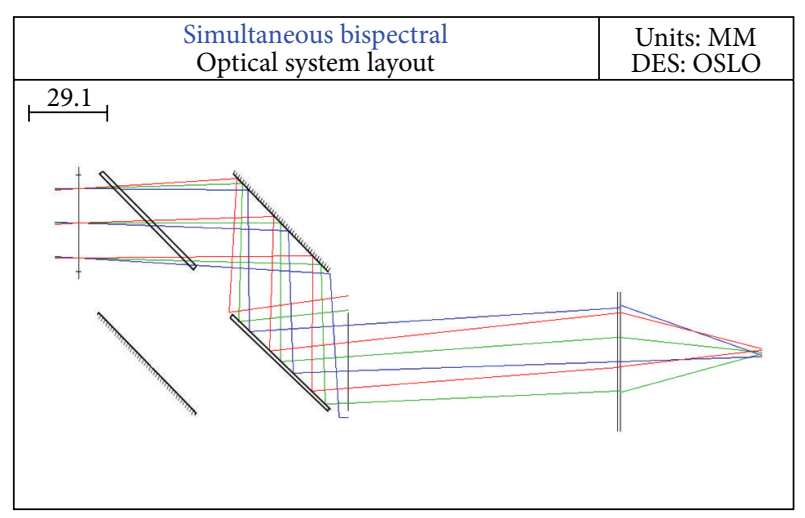

(a) Upper channel

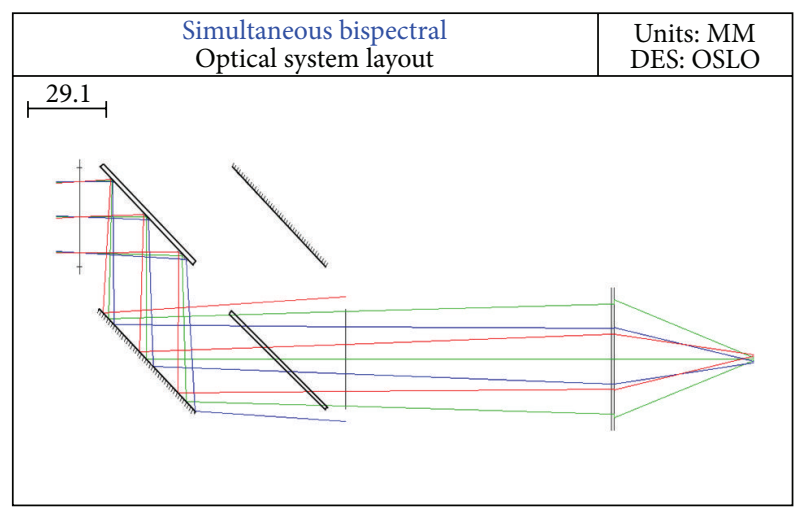

(b) Lower channel

FIGURE 3: Separate imaging is accomplished through upper (a) and lower (b) channels. Each beam goes through the same path: one reflection at a beam splitter, one transmission at a beam splitter, and one reflection at a 45-degree mirror.

Next we examine the requirements for the optical system design and we look at several possible layouts. Then, we elaborate on the most promising choice for its implementation for our applications.

3.1. Optical System Requirements. The requirements on the optical system are derived from the layout and performance proposed to measure the efficiency of the combustion process. This is achieved by taking the ratio of the radiation emitted by the object/flame. The radiation is split into two beams, each with its own optical system. Each beam is transmitted through the selective filter. A rapid IR camera with a large number of pixels is suitable as a focal plane to incorporate as one of the principal components into the radiometric chain. There are great advantages of using one detecting surface for two paths, because it is only necessary to characterize and calibrate one detector. Furthermore, the camera signal post-processing is same for all pixels. Methods have been established long time ago to pick up the signal from the corresponding pixels [17] and to perform image processing.

Two imaging channels for each spectral band need to be laid out, such that the flame is imaged on the adjacent area on the camera focal plane with minimal and equal amount of aberrations. Due to imaging with two different wavelengths, the effects of the chromatic aberrations must be minimized. Then the radiation that is emitted from the same pixel on the flame projected on the object plane is imaged on the same area on two adjacent images. This requires two optical systems with the axis of one slightly inclined with respect to that of the other.

3.2. Candidate Optical Layouts. We examined three candidate optical systems as possible layouts for two-path IR imaging on adjacent locations on the focal plane. The optical systems incorporating two identical paths in the visible light have been well understood and described for their widespread incorporation into interferometric systems [18]. The situation is made somewhat more difficult in the IR because the coating materials do not have the same performance as in the visible. The alignment challenges are somewhat exacerbated due to the absence of visual information. A high throughput is not a requirement due to large amount of photon generation in the combustion. However, the amount of IR light that is not the signal contributes to the invisible noise that may interfere with the alignment and detected signal in the focal plane, as a stray light. All the design work was performed on OSLO. Figure 2 presents three layouts that were studied as to their overall feasibility [19]. Part (a) shows the two mirror, two beam splitter configuration; part (b) presents two-mirror, one-beam-splitter layout; and part (c) features a four-mirror proposal.

The first configuration (Figure 2(a)) is a symmetrical arrangement of two mirrors and two beam splitters. Symmetry is an advantage of this layout, including that the optical path is the same for both trajectories. The filters can be easily inserted. The same scene is split in two images. This layout transmits twenty five percent of the incoming light from the scene for each image. The second configuration (Figure 2(b)) consists of two mirrors and one beam splitter. This is the simplest of the three configurations but it has very limited physical space to accommodate the filters. This configuration transmits fifty percent of the incoming light. It has also different path length for each trajectory, so even in the bestfocus plane it presents the worse performance. The third configuration (Figure 2(c)) is a symmetrical layout that uses no beam splitters, only mirrors, but each image is seen from a different perspective. We selected the first configuration because it is symmetrical, it has space to accommodate the filters, it is a layout with superior optical performance, and it is the only one that assures the same amplification and perspective of the inspected object for both images.

3.3. Optimal Layout of the Optical System. We selected a two-mirror, two-beam-splitter optical layout, indicated in Figure 3, similar to the traditional Mach-Zehnder interferometric configuration, but used in imaging mode [20]. The well-accepted beauty of this layout is its compactness, allowing the portability of this instrument for in-the-field, in situ measurements. The optical system layout for the upper channel is indicated in part (a), with the lower channel shown 


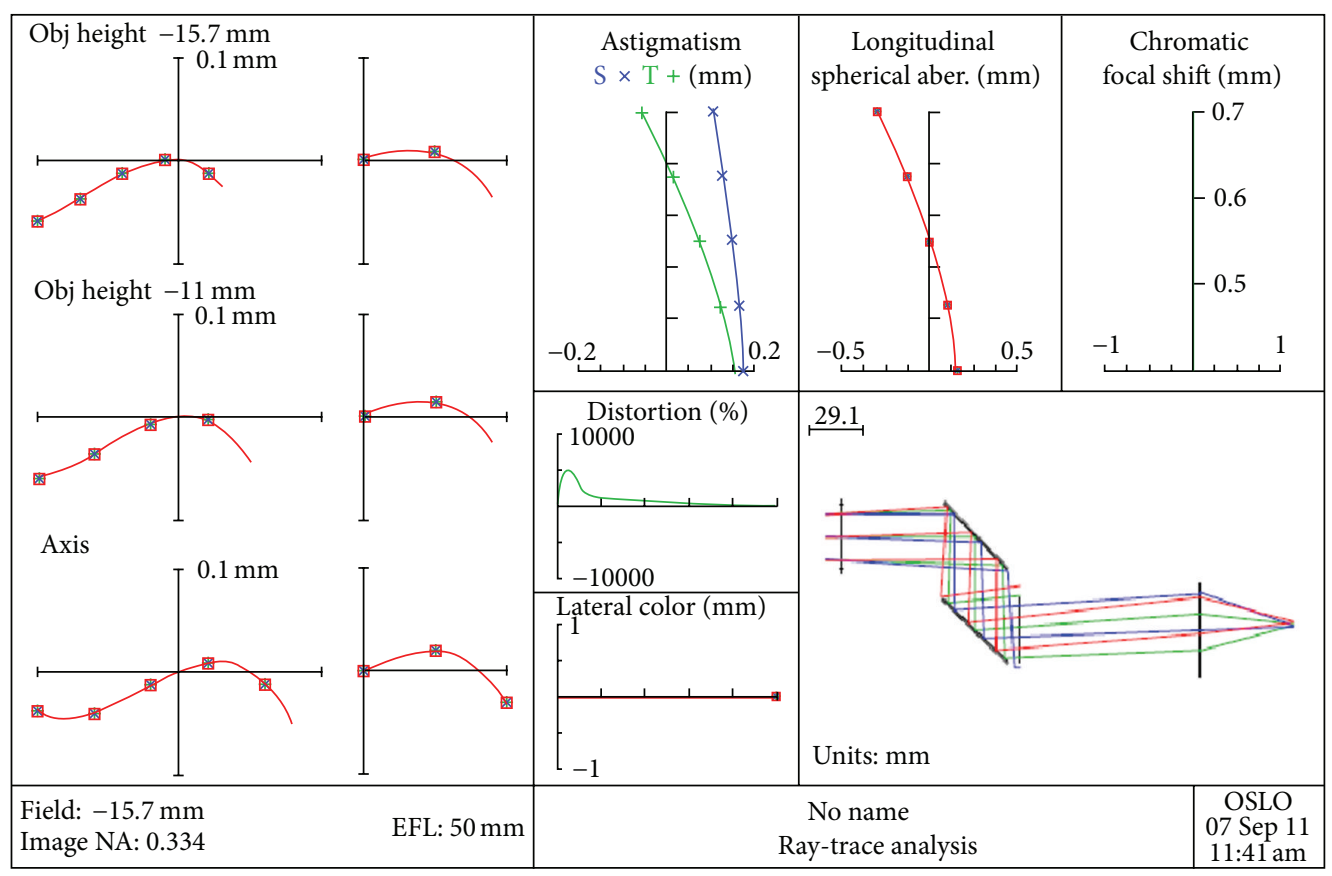

(a) Upper arm

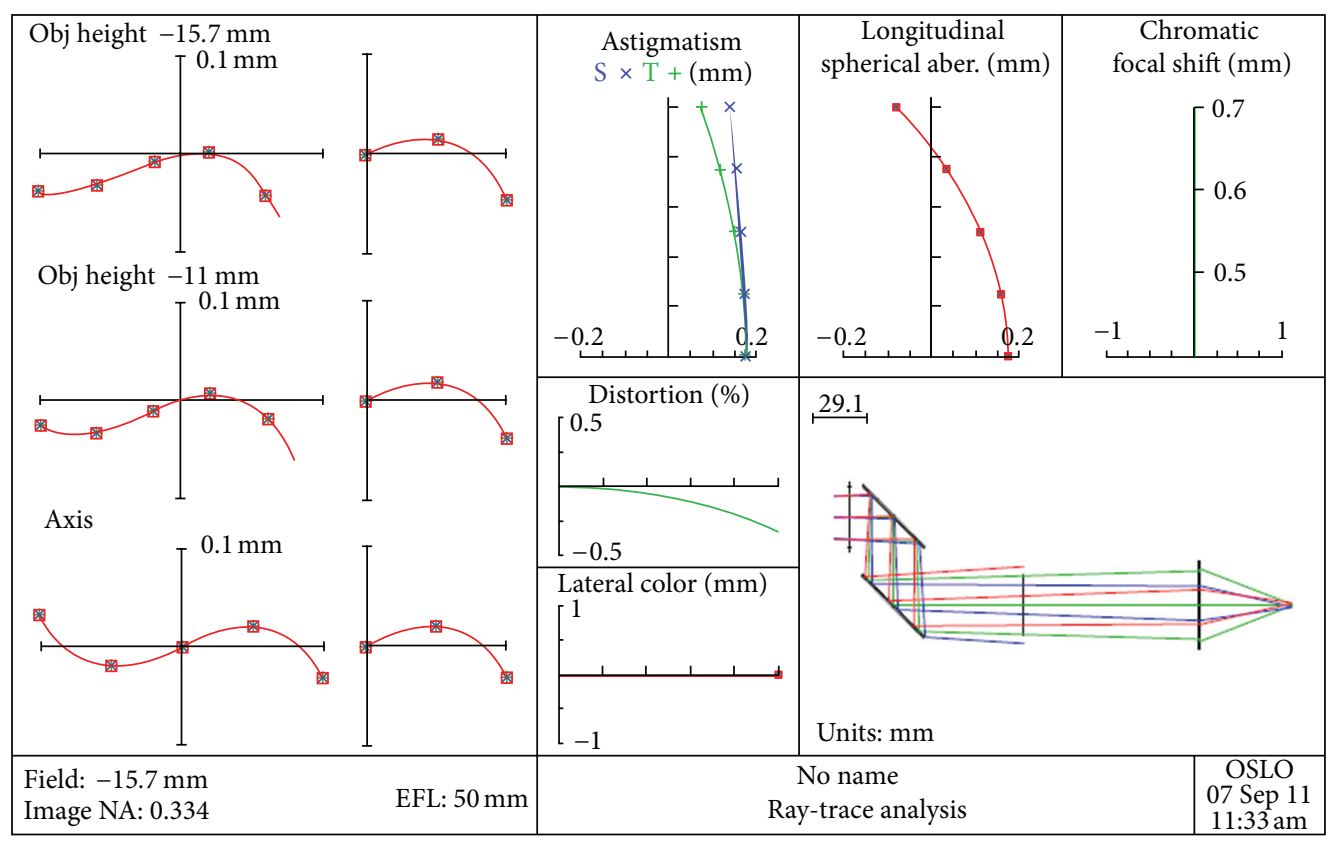

(b) Lower arm

FIGURE 4: Results of the exact ray-trace analysis, obtained with OSLO: (a) upper arm, (b) lower arm.

in part (b). The figure includes the ray tracing on axis and off axis. It shows the advantages that the symmetry of the optical system represents for the formation of both images from the same object. The optical axes of two channels are at a small angle with respect to each other to allow side-toside positioning of two images in the focal plane. The image distortion is less than $0.5 \%$ (cannot be noticed with the bare eye) and can be easily determined and corrected. The results of the ray-trace analysis performed with OSLO are presented in Figure 4, the upper arm in part (a), and the lower arm in part (b). It can be seen in the ray trace analysis that the aberrations are of the same order of magnitude for each arm; this is because of the symmetry of the system. Additionally, given that symmetry, in both cases the aberrations are quite 
similar. The transversal aberration shows mainly a minor amount of astigmatism at the best focus, very similar on axis and off axis. Chromatic aberration is insignificant, because of the reflective surfaces, and there is only a minor contribution in the refraction of the beam splitter. Once more, the chromatic aberration is very similar for both arms, given the symmetry.

The image quality in general does not represent a significant concern for IR imaging due to its long wavelengths: the diffraction limit spot diameter is usually larger than the spot spread due to the aberrations. In this particular case, it is imperative that the performances of two parallel channels be identical because imaging in different IR wavelength (bands) may actually result in longitudinally displaced focal planes. This would be detrimental to the accuracy of the technique given that we actually count the number of photons incident from one pixel projected on the object onto two detector pixels. If the imaging is not faithful, different image pixels do not correspond to each other and the object pixel may be compressed along the optical axis. We simulate the imaging of the object screen to two images at the same focal plane location, along different arms. Upon examining Figure 5 we can deduce that images of a square mesh from both channels are equally well preserved. This results in excellent pixel-topixel correspondence between the upper and lower arms. Simultaneous, two-channel imaging through both arms is indicated in Figure 6, in a compact, two-mirror, two-beam slitter IR optical system.

3.4. Spatially Displaced Bispectral Images. We incorporate this optical system into an instrument, similar to the MachZehnder shearing interferometer, with the modifications indicated in Figure 1. We chose this particular arrangement, because both optical paths are sufficiently similar to allow pixel-by-pixel comparison of two images. Otherwise, the bispectral images may appear in focus in displaced focal planes and exhibit different magnification. The differences between the bispectral and the interferometric imaging are that in the former the two beams and images are placed on different pixels of the detector array, and that there is no need for coherence. The symmetry of the optical system in two arms is also helpful in decreasing the vignetting after the data has been processed. Likewise, the pixel-by-pixel comparison is facilitated when both images travel through nearly identical optical systems.

The object of interest, referred in Figure 1 as a specimen, is placed on the left side of the optical system. The IR amplitudedividing beam splitter transmits $50 \%$ of the collected radiation and reflects the other $50 \%$ of light. This beam splitter is placed at 45 degrees with respect to the optical axis, defined as the line connecting the center of the object with the center of the detector array, excluding the effects of beam directionturning mirrors.

Mirrors M1 and M2 are placed at an angle of 45 degrees in each beam path to change the beam direction of each beam by 90 degrees. Mirrors MA and MB are standard silvercoated mirrors. BSA and BSB are MWIR 50/50 beam splitters. Inside each optical path we insert an IR band-pass filter

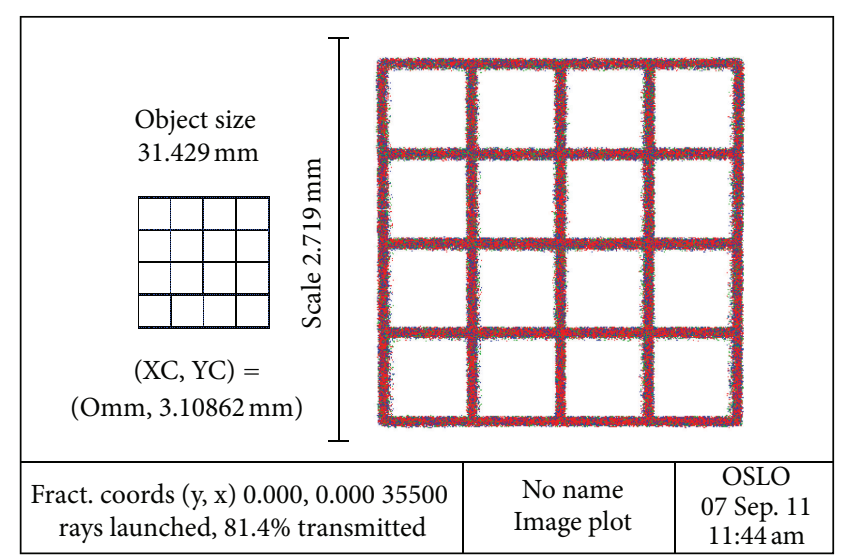

(a) Upper arm

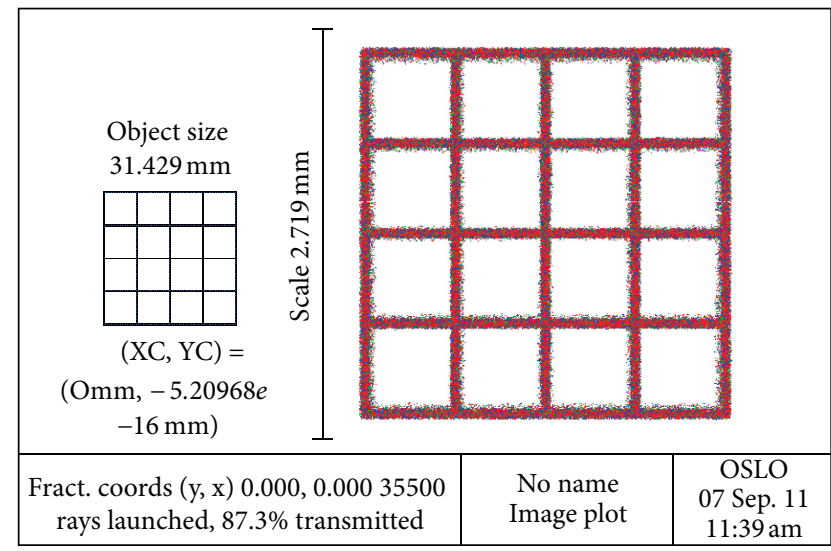

(b) Lower arm

FIGURE 5: Simulation results of chromatic image formation: (a) upper arm, (b) lower arm.

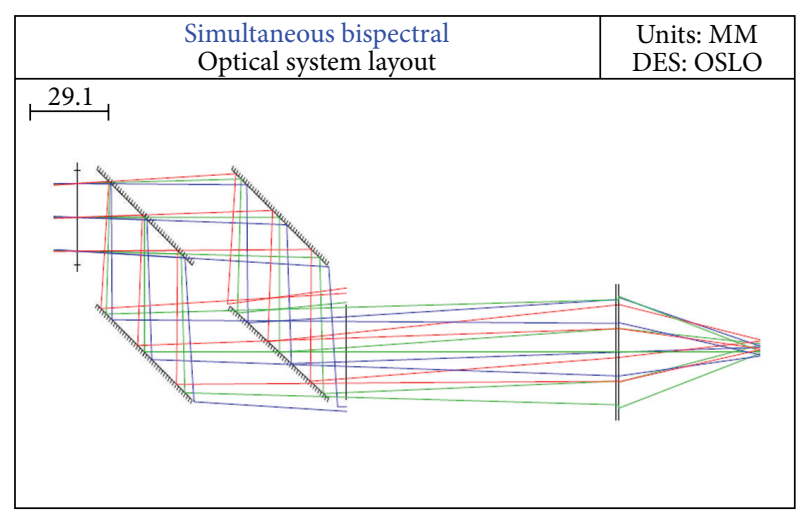

FIGURE 6: Exact ray tracing through the optical system that performs simultaneous bispectral imaging.

(FA and FB) that may be custom-selected for the specific application. The beams are identical just before they are incident on their respective filters. After they pass through the filters, the two beams are spectrally modified to transmit two spectral images, corresponding to the filter transmission 


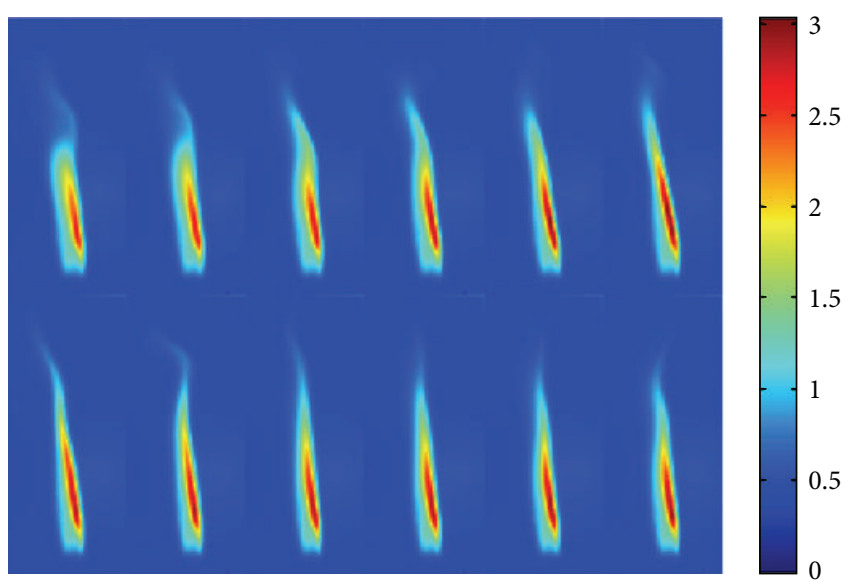

FIGURE 7: Twelve representative images illustrating the relative spatial combustion efficiency for a low-oxygenation flame during the initial one second after ignition, after the beginning of the combustion, from $t=0 \mathrm{sec}$ (top left image) to $t=1 \mathrm{sec}$ (bottom right image). In the black-and-white rendition, the dark lines inside the flames indicate ratio of about three between the amounts of carbon monoxide to that of dioxide. The inside region does not participate in an effective combustion, generating mostly carbon monoxide.

function. Each image is incident on the second amplitudedividing beam splitter that is positioned at a small angle $\Delta$ with respect to 45 degree line; say at 40 degrees with respect to the horizontal. The beam that is incident on the beam splitter from below reflects onto the detector to the right of the beam that is transmitted arriving from the left. We spatially separate the positions of the images on the focal plane of the IR camera, tagging each image by its location. It is also possible to modify the angles of the other optical components (MA, $\mathrm{MB}$, and $\mathrm{BSA}$ ) to achieve a wider image separation. Each beam, upon its incidence on the focal plane of the camera, forms a filtered in-focus image. All the distances are kept small to facilitate the component alignment, to maximize the optical throughput and to keep the instrument compact.

In our experimental setup, we integrate the IR camera Silver, manufactured by Cedip. It operates in the [3.6 $\mu \mathrm{m}-$ $5.1 \mu \mathrm{m}]$ spectral interval. We incorporate advanced electronic features of this camera, allowing them to drive some of the other system parameters. The Silver model includes a motorized focus and an internal filter wheel. The former restricts the space inside the camera enclosure, so we implement the external filter arrangement with a separate path for each filter. The simultaneous image taking guarantees identical exposures even for a rapidly changing object.

In the field of spectroscopy, the flame is characterized with chemical by-products, with specific spectral lines. Their relative magnitude indicates the degree of efficiency of fuel utilization. Thus, our "parallel-path" optical systems allow us to identify and quantify the by-products to measure the efficiency of the fuel consumption at a very rapid rate. A single camera assures simultaneous detection of two images during the time interval equal to about one millisecond of the detector-integration time.

\section{Bispectral Imaging Applied to Combustion}

The development of this technique has been motivated by the need to study the combustion efficiency of the ignition process and evolution. The ideal combustion of fossil fuels produces only water vapor and carbon dioxide. As the combustion efficiency deteriorates, amounts of intermediate species increase, in particular, that of the carbon monoxide. Carbon dioxide has a strong emission band centered on $4.3 \mu \mathrm{m}$, while carbon monoxide has one centered on $4.8 \mu \mathrm{m}$ and several lines near $4.66 \mu \mathrm{m}$ [21]. On each image detected on the camera focal plane, the left flame, from the viewer's point of view, is filtered using a $4.66 \mu \mathrm{m}$ centered filter B $(0.164 \mu \mathrm{m}$ full width at half maximum, FWHM, transmission band) to monitor the carbon monoxide emission band. The right flame is filtered with a $4.3 \mu \mathrm{m}$ centered filter $\mathrm{A}(0.181 \mu \mathrm{m}$, FWHM,transmission band), corresponding to the carbon dioxide emission band.

We study a butane flame, generated by a Bunsen burner, controlling the amounts of oxygen. We display the representative results in Figure 7, demonstrating the feasibility of this technique. It illustrates twelve bispectral images of a small flame, generated by a low-oxygenation butane burner, during the first second. We obtain them at the rate of 1200 per second, by dividing incidence through one filtered image by the other, pixel by pixel. Appreciable signal at $4.66 \mu \mathrm{m}$ filter B indicates carbon monoxide.

The radiation carrying useful flame information is incident on and detected by the central part of the detector array. This permits us to employ image subframing features of the Silver camera model. With the limitation of the pixel integration time, the experimentalist may capture up to 1,200 frames per second. We operate the camera in subframing mode in order to obtain 1200 frames per second, with a resolution of $160 \times 128$ pixels. Depending on the specific application, it would be necessary to have a higher spatial resolution, possible by reducing the frame rate.

\section{Summary}

In this work we presented the design of an optical system for bispectral imaging with inclined axes, using a single camera. It has been incorporated into a versatile experimental setup to measure the beam transmission through two channels, allowing the incorporation of spectral filters to verify the presence of specific spectral components and to quantify them. Its excellent performance allowed precise imaging of one projected pixel on the object plane to two pixels on the image plane with the same and very small image deterioration.

The main advantage of bispectral imaging over other techniques is that it allows capturing two simultaneous images of a sample, at a high rate. Both (flame) images are captured on the same focal plane and on the same frame. Over 1000 frames per second may be recorded when the object is of adequate size to permit subframing. After performing pixelby-pixel ratio of both channels for the same projected pixel, a determination about the efficiency of the combustion process may be made. 
Experimental work is in progress to determine the efficiency through the volume corresponding to each pixel as a function of time for different combustion conditions. We are particularly interested in understanding the nature of the small instabilities that are colloquially described as scintillations on the top of the flame.

\section{References}

[1] M. Strojnik, G. Paez, and C. Vasquez-Jaccaud, "Flame evaluation during first second after ignition in a gas stove," in Infrared Spaceborne Remote Sensing and Instrumentation XVI, vol. 7082 of Proceedings of SPIE, pp. 70820N-1-70820N-7, SPIE, Bellingham, Wash, USA, 2008.

[2] G. Paez and M. Strojnik, "Interferometric characterization of flames," in Unconventional Imaging II, Proceedings of SPIE, pp. 6307-635, SPIE, Bellingham, Wash, USA, 2006.

[3] M. Strojnik, G. Paez, and J. C. . Granados, "Flame thermometry," in Unconventional Imaging II, vol. 6307 of Proceedings of SPIE, pp. 63070L1-63070L8, SPIE, Bellingham, Wash, USA, 2006.

[4] B. García-Lorenzo, J. J. Fuensalida, M. A. C. RodríguezHernández et al., "EDiFiSE: equalized and diffraction-limited field spectrograph experiment," in Ground-based and Airborne Instrumentation for Astronomy II, 70144B, vol. 7014 of Proceedings of SPIE, SPIE, Bellingham, Wash, USA, 2008.

[5] T. Wilson and C. Davis, "Hyperspectral Remote Sensing Technology (HRST) program and the Naval EarthMap Observer (NEMO) satellite," in Conference on Infrared Spaceborne Remote Sensing VI, vol. 3437 of Proceedings of SPIE, pp. 2-10, SPIE, Bellingham, Wash, USA, July 1998.

[6] G. S. Libonate, B. Denley, E. E. Krueger, J. H. Rutter, J. A. Stobie, and C. L. Terzis, "Development status of the AIRS IR focal plane assembly," in Infrared Spaceborne Remote Sensing V, vol. 3122 of Proceedings of SPIE, pp. 174-184, Bellingham, Wash, USA.

[7] M. S. Scholl, Y. Wang, J. E. Randolph, and J. A. Ayon, "Site certification imaging sensor for mars exploration," Optical Engineering, vol. 30, no. 5, pp. 590-597, 1991.

[8] J. Castrellon, G. Paez, and M. Strojnik, "Remote temperature sensor employing erbium-doped silica fiber," Infrared Physics and Technology, vol. 43, no. 3-5, pp. 219-222, 2002.

[9] A. Aranda, M. Strojnik, G. Paez, and G. Moreno, "Twowavelength differential thermometry for microscopic extended source," Infrared Physics and Technology, vol. 49, no. 3, pp. 205209, 2007.

[10] C. Vazquez-Jaccaud, G. Paez, and M. Strojnik, "Wavelength selection method with standard deviation: application to pulse oximetry," Annals of Biomedical Engineering, vol. 39, no. 7, pp. 1994-2009, 2011.

[11] P. Mitra, S. L. Barnes, F. C. Case et al., "MOCVD of bandgapengineered $\mathrm{HgCdTe}$ p-n-N-P dual-band infrared detector arrays," Journal of Electronic Materials, vol. 26, no. 6, pp. 482487, 1997.

[12] S. D. Gunapala, S. V. Bandara, J. K. Liu et al., "1024 × 1024 Format pixel co-located simultaneously readable dual-band QWIP focal plane," Infrared Physics and Technology, vol. 52, no. 6, pp. 395-398, 2009.

[13] L. N. Phong and I. Shih, "Optically coated dual sensors for wavelength detection," in Infrared Technology and Applications XXIII, vol. 3061 of Proceedings of SPIE, pp. 172-184, SPIE, Bellingham, Wash, USA, 1997.
[14] R. P. Jones, D. P. Jones, and M. Lidwell, "Dual waveband optics for the visible and mid-infrared waveband infrared," in Infrared Technology and Applications XXIII, vol. 3061 of Proceedings of SPIE, pp. 388-395, SPIE, Bellingham, Wash, USA, 1997.

[15] K. Kinosita, H. Itoh, S. Ishiwata, K. Hirano, T. Nishizaka, and T. Hayakawa, "Dual-view microscopy with a single camera: realtime imaging of molecular orientations and calcium," Journal of Cell Biology, vol. 115, no. 1, pp. 67-73, 1991.

[16] G. Garcia-Torales, M. Strojnik, and G. Paez, "Risley prisms to control wave-front tilt and displacement in a vectorial shearing interferometer," Applied Optics, vol. 41, no. 7, pp. 1380-1384, 2002.

[17] M. Strojnik, "From radiometric and thermographic techniques to characterize an IR target to temperature monitoring of a diabetic foot," in Tribute to William Wolfe, Proceedings of SPIE, pp. 8483-8487, SPIE, Bellingham, Wash, USA, 2012.

[18] I. Moreno, G. Paez, and M. Strojnik, "Dove prism with increased throughput for implementation in a rotational-shearing interferometer," Applied Optics, vol. 42, no. 22, pp. 4514-4521, 2003.

[19] H. Gross, F. Blechinger, and B. Achtner, Handbook of Optical Systems, Survey of Optical Instruments, vol. 402, Wiley-VCH, 2008.

[20] C. Ramirez and M. Strojnik, "Performance evaluation of a wavefront displacement system for vectorial shearing interferometer," Optics Communications, vol. 281, no. 3, pp. 347-355, 2008.

[21] S. Wedemeyer-Böhm, I. Kamp, J. Bruls, and B. Freytag, "Carbon monoxide in the solar atmosphere," Astronomy \& Astrophysics, vol. 438, pp. 1043-1057, 2005. 

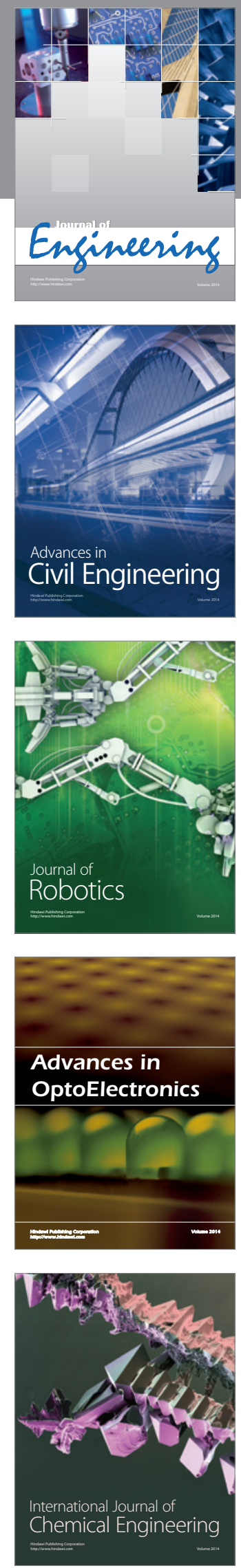

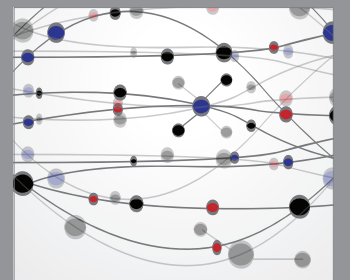

The Scientific World Journal
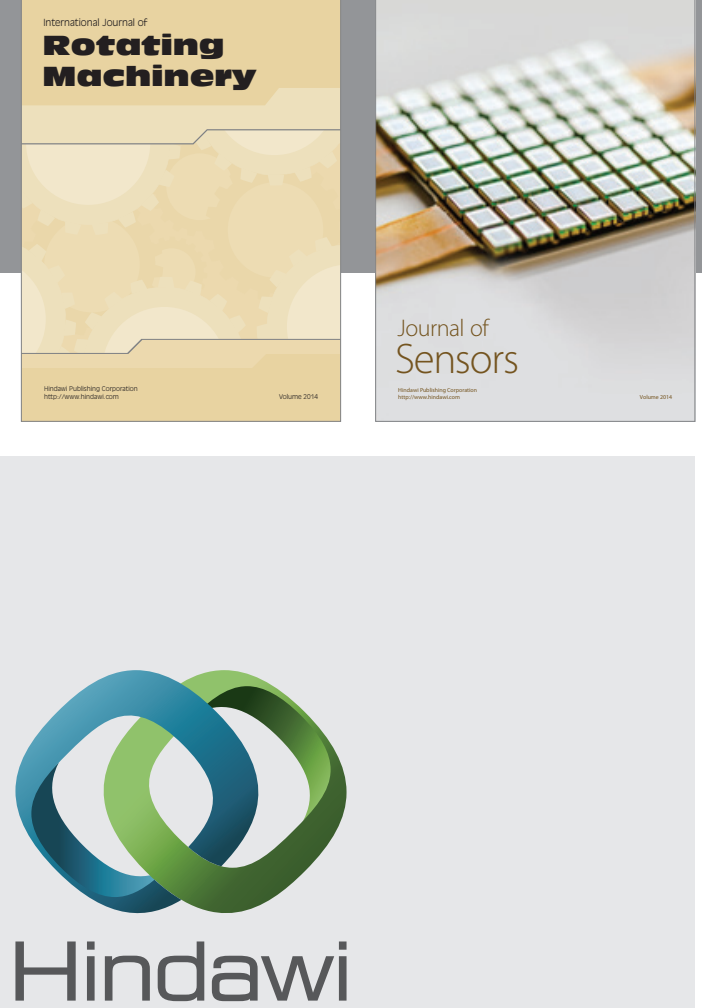

Submit your manuscripts at http://www.hindawi.com
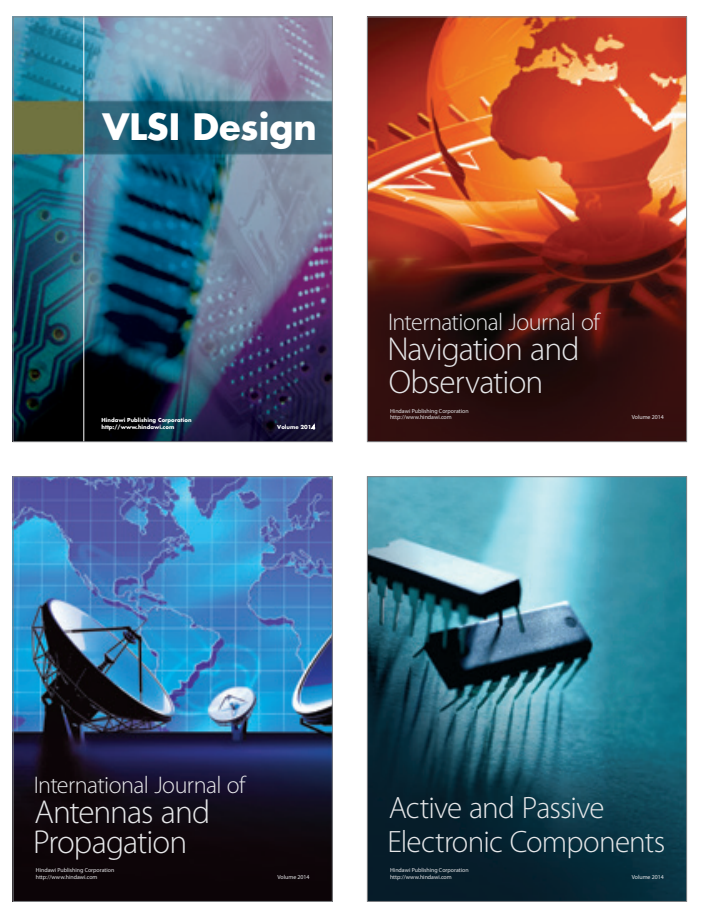
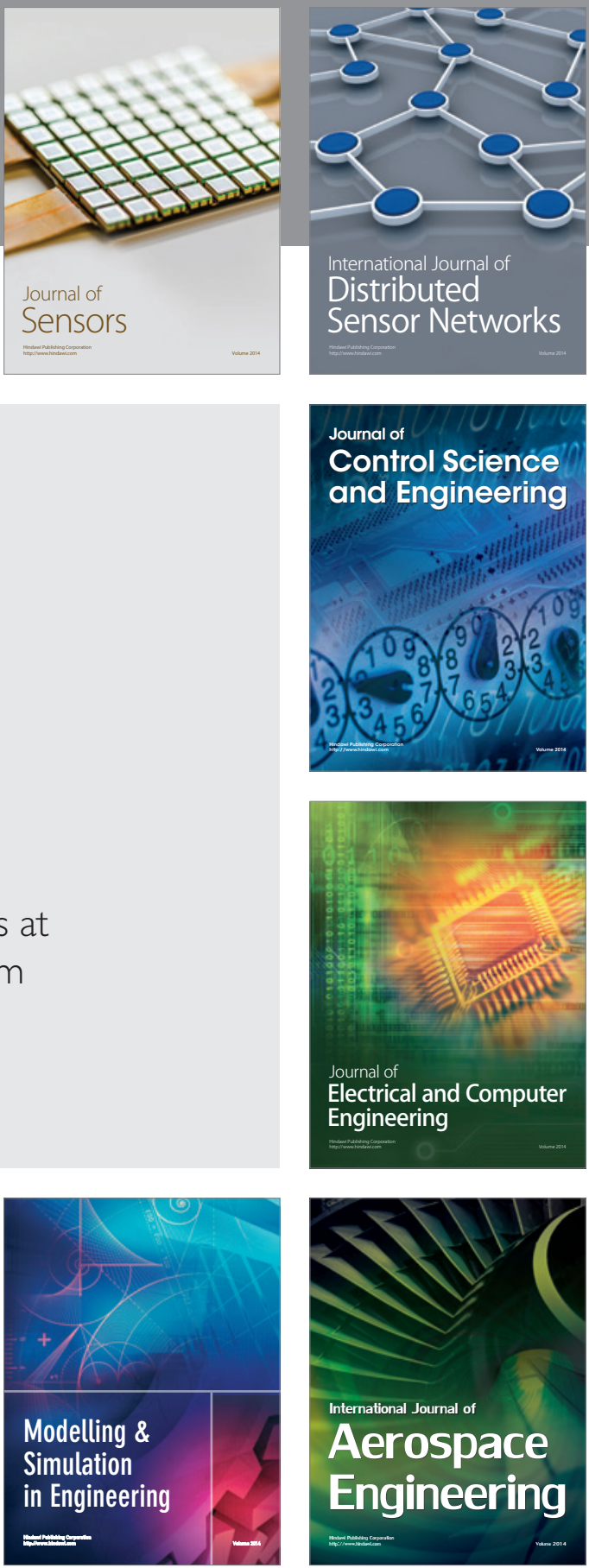

Journal of

Control Science

and Engineering
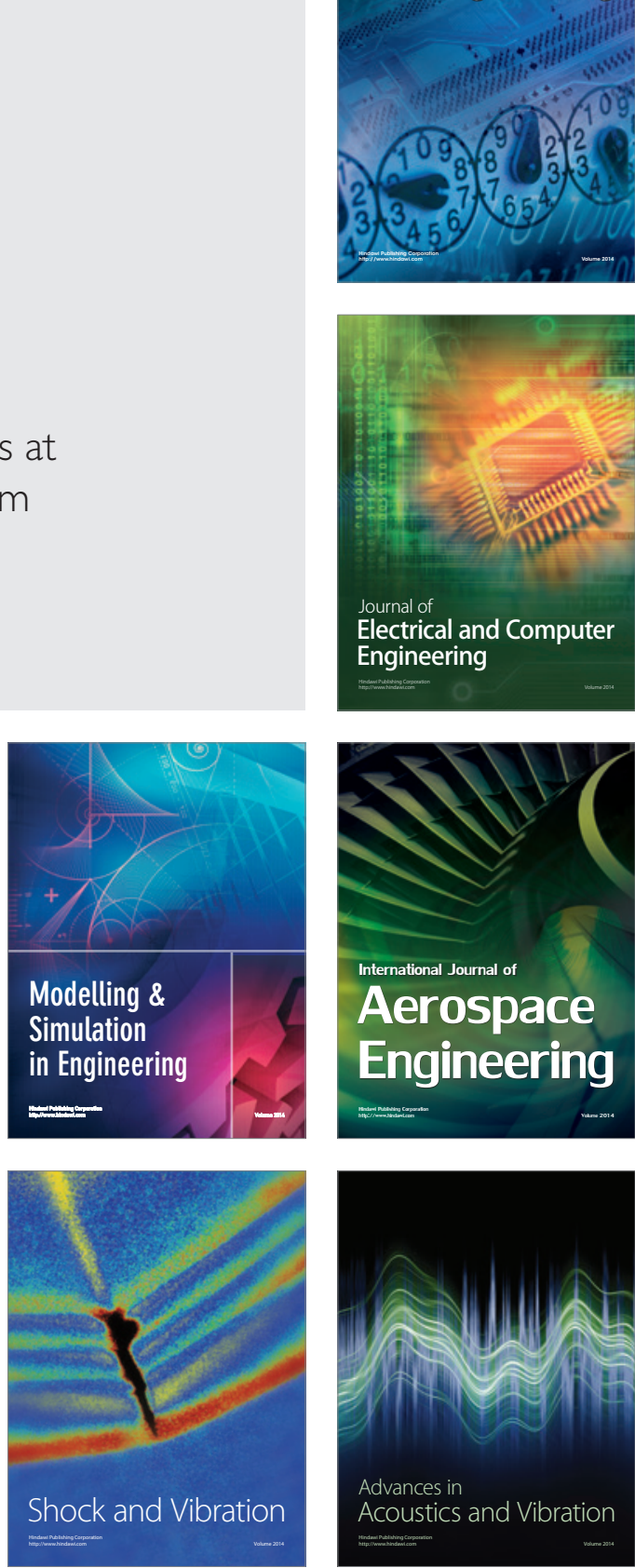